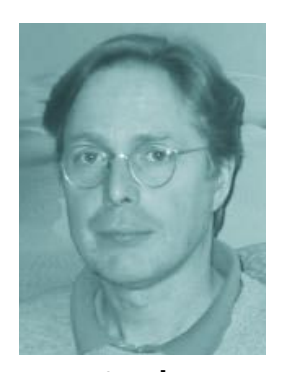

Uwe Kinzel

\section{Medikamente und ihre Nebenwirkungen auf die Sexualität}

\author{
Uwe Kinzel, Wolfgang Weig \\ Niedersächsisches Landeskrankenhauses Osnabrück
}

psychoneuro 2003; 30 (1): 53-56
S exuelle Nebenwirkungen von Medikamenten sind in den letzten Jahren vermehrt in den Blick gleichermaßen von Behandlern und Pharmaindustrie gerückt. Früher wurden sie nicht berücksichtigt, nicht untersucht, diskutiert. Heute sind fehlende sexuelle Nebenwirkungen von Medikamenten Werbemittel, die zur Zeit durch die Hersteller von Pharmaka gerne in den Vordergrund für die Verordner gerückt werden.

Auch in der Literatur zur Sexualmedizin werden sexuelle Nebenwirkungen von Medikamenten vermehrt berücksichtigt. Jedoch sind ausführliche Überblicksreferate selten. Vergleicht man Listen von Medikamenten, die derartige Nebenwirkungen haben, so wird deutlich, dass vor rund 20 Jahren 60 Präparate und Medikamentengruppen (1) zu finden waren, heute sind es annähernd 200 (2). Dem hat auch die Psychiatrie Rechnung getragen. Die Amerikanische Psychiatriegesellschaft APA führte im DSM-IV die diagnostische Kategorie: Substanzinduzierte sexuelle Störung ein. (3)

Die Definition lautet folgendermaßen:

- Kriterium a: Eine klinisch bedeutsame sexuelle Funktionsstörung, die zu deutlichem Leiden oder zwischenmenschlichen Schwierigkeiten führt, ist nachweisbar.

Verschiedene Medikamentengruppen stehen in Zusammenhang mit sexuellen Dysfunktionen. Vor allem kardio-vaskuläre und psychotrope Substanzen, aber auch andere Medikamente beeinflussen verschiedene Hormone und können so sexuelle Dysfunktionen hervorrufen. In diesem Beitrag werden die verschiedenen Substanzen und ihre Nebenwirkungen diskutiert sowie therapeutische Optionen vorgeschlagen.

- Kriterium b: Die Vorgeschichte, die körperliche Untersuchung oder Laborbefunde haben den Nachweis dafür erbracht, dass eine sexuelle Funktionsstörung vollständig durch die Substanzeinnahme erklärt wird. Eingenommene Medikamente stehen im ätiologischen Zusammenhang mit dem Störungsbild.

- Kriterium c: Das Störungsbild kann nicht besser durch eine sexuelle Funktionsstörung erklärt werden, die nicht substanzinduziert ist.

In dieser Definition sind bereits Hinweise auf das Vorgehen bei Verdacht auf eine medikamentös induzierte sexuelle Störung zu erkennen. Es müssen andere Ursachen ausgeschlossen werden, wenn ein zeitlicher Zusammenhang zwischen Medikation und Auftreten der Störung vorhanden ist.

1. Kann die Störung ein Symptom der Erkrankung sein, wie z.B. eine Erektionsstörung als erstes Symptom einer depressiven Episode?

2. Bei ungenauer Anamneseerhebung kann die sexuelle Störung schon vor Gabe der Medikation bestanden haben.

3. Die sexuelle Störung kann psychoreaktiv auf die Erkrankung entstanden sein.

Bei einer sehr spezifischen Störung, also bei Fehlen das Allgemeinbefinden beeinträchtigender Nebenwirkungen und der Beeinträchtigung einer speziellen sexuellen Funktion, sollte dies ein Hinweis auf Arzneimittelverursachung sein (4).

Eine weitere Orientierung gibt der Blick auf Pharmaka, die häufig verordnet werden und Nebenwirkungen auf sexuelle Funktionen haben:

- Kardio-vaskulär wirksame Medikamente

- Psychopharmaka

- Pharmaka mit endokrinen Wirkungen.

Vieles spricht dafür, dass die meisten Substanzen bei beiden $\mathrm{Ge}-$ schlechtern sexuelle Störungen bedingen können (siehe auch Tab. 1). Untersucht sind jedoch bisher überwiegend männliche Probanden. Die Ursache dafür, dass nur geringe Zahlen weiblicher Probanden in klini- 


\section{Tab. 1 Effekte der Neurotransmitter auf die sexuelle Funktion bei Mann und Frau}

Dopamin: Führt bei Freisetzung zu Erektion, bei Frauen zu sexueller Erregung (arousal) und Lubrikation.

5-Hydroxytryptamin: Führt bei Freisetzung zu verzögertem Einsetzen von Ejakulation und Orgasmus.

Noradrenalin: Führt über Beeinflussung von Beta-Rezeptoren zur Erektion, über die Bindung an Alpha1-Rezeptoren zu Ejakulation und Orgasmus.

Acetylcholin: Wird bei Frauen ein modulierender Effekt auf hypothalamische M2-Rezeptoren gegenüber Östrogen zugeschrieben.

\section{Tab. 2 Therapeutische Optionen bei sexuellen Nebenwirkungen unter Antidepressiva}

1. Dosisreduktion: Bei Antidepressiva ist die Reduktion der Dosis problematisch, weil die Dosis, die zur Remission führte z.B. für die major depression in der Remission oder in der Rezidivprophylaxe beibehalten werden sollte. Die Dauer sollte zumindest ein Jahr betragen.

2. Medikamentenpause: Bei den SSRI, z.B. Paroxetin und Sertralin, eine durchaus mögliche Alternative. Allerdings nicht bei Fluoxetin infolge der langen Halbwertzeit. Eine Medikamentenpause von 2 bis 3 Tagen z.B. freitags bis sonntags ist möglich und damit eine deutliche Reduktion von Nebenwirkungen. Ein Problem bei dieser Vorgehensweise ergibt sich darin, dass der Patient die Idee entwickeln könnte, das Medikament sei nicht länger notwendig, so dass er die weitere Einnahme unterbricht und somit ein Rezidiv der depressiven Erkrankung riskiert.

\section{Wechsel des Antidepressivums:}

Wechsel auf die sehr nebenwirkungsarmen Präparate Mirtazapin und Moclobemid.

\section{Zusatzmedikation:}

Amantadin: Durch Antidepressiva ausgelöste Erektionsstörung: $100 \mathrm{mg} 2$ bis 3 Stunden vor sexueller Aktivität bewirkt eine deutliche Besserung von Erektionsstörung und Anorgasmie (9).

Betanechol: $25 \mathrm{mg} 1$ bis 2 Stunden vor sexueller Aktivität einzunehmen gegen Erektionsstörungen bei Behandlung mit Antidepressiva.

Carbachol: gegen Erektionsstörungen, Ejakulations- und Orgasmusstörungen, 2 mg, einzunehmen 1 bis 2 Stunden vor sexueller Aktivität.

Cyproheptadin: Bei Behandlung mit Serotoninwiederaufnahmehemmern und dadurch aufgetretener Erektionsstörung und Anorgasmie eignet sich die Zugabe von $4 \mathrm{mg}$ Cyproheptadin, ein bis zwei Stunden vor sexueller Aktivität (8).

Yohimbin: $5 \mathrm{mg} 1$ bis 2 Tabletten dreimal pro Tag über 4 Wochen kurmäßig eingesetzt erwiesen sich als wirksam als Zusatzmedikation bei SSRI-induzierten Erektionsstörungen (10). sche Medikamentenstudien aufgenommen wurden, sind vielfältig. Die Pharmaindustrie schützt sich damit vor möglichen Regressen bei schwangeren Studienteilnehmerinnen sowie gegen die Argumentation, dass die hormonelle Situation der Frauen Einfluss auf die Wirkung des Pharmakons genommen hat. $\mathrm{Zu}$ mindest erscheint es durch diesen Umstand so, als würden viele Arzneimittel nur die Sexualität des Mannes beeinträchtigen.

\section{Medikamentengruppen \\ Kardio-vaskulär wirksame Medikamente}

Diuretika: Tiaziddiuretika verursachen gelegentlich zentralnervöse, wie auch sexuelle Störungen. Kombinationstherapie mit Beta-Blockern führen nach einer Literaturdurchsicht zu einer erhöhten Rate an sexuellen Dysfunktionen. Insbesondere Propranolol und Diuretika verursachen derartige Wirkungen. Aldosteronantagonisten, wie z.B. Spironolacton bewirken durch ihre Steroidstruktur und die Störung der Testosteronsynthese ausgeprägte sexuelle Dysfunktionen hier insbesondere Störung von Libido und Erektion.

Sympathikolytika: wie z.B. Guanethidin wirken antihypertensiv durch Herabsetzung des erhöhten Sympathikotonus. Abhängig von der Ausgangslage können sie die Erregungsinitiierung und den Orgasmus beeinträchtigen. Adrenerge Agonisten an Autorezeptoren wie z.B. Clonidin (Catapresan ${ }^{\circledR}$ ) bewirken eine Hemmung der zentralen Sympathikusefferenz. Diese Wirkung zeigt ebenfalls Methyl-Dopa, das die sexuelle Funktionsfähigkeit beeinträchtigt, insbesondere Libido und Erektion.

Beta-Adrenozeptorantagonisten (Beta-Blocker): Die noch nicht klar definierten Auswirkungen auf die sexuelle Funktion (insbesondere Erektionsstörungen, Verminderung des Ejakulatvolumens und retrograde Ejakulation, wie auch Libidostörung) dürften teils zentral, teils vaskulär zustande kommen.

Vasodilatatoren, wie Minoxidil und Hydralazin, scheinen keine besondere Nebenwirkungen aufzuweisen, allerdings wird gelegentlich Priapismus beobachtet.

ACE-Hemmer, wie Enalapril und Captopril, bedingen relativ selten sexuelle Nebenwirkungen, wie Erektionsstörungen (4). Als günstig erwiesen sich in kontrollierten Studien insbesondere Captopril und der Angiotensin2-Rezeptor-Blocker Losartan. Dieses Präparat führte nach einer neueren US-Studie sogar zu einer Verbesserung von Erektionsstörungen.

\section{Psychopharmaka}

Antidepressiva: Insbesondere seit Einführung von Prozac ${ }^{\mathrm{TM}}$, bzw. des in Deutschland unter dem Namen Fluctin ${ }^{\circledR}$ bekannt gewordenen Antidepressivums, das seither in den Medien als „Happy-Pill“ bezeichnet wurde, kann mit Blick auf sexuelle Nebenwirkungen dieser Präparategruppe keine Fröhlichkeit aufkommen. Es werden zwar unter Fluoxetin und Fluvoxamin, wie auch unter Bupropion in Einzellfallbeschreibungen rezidivierende Spontanorgasmen mitgeteilt, ebenfalls Orgasmen, die mit Gähnen assoziiert waren (5), jedoch überwiegen negative Wirkungen, wie z.B. Erektionsstörungen und Ejakulationsverzögerungen. Warum diese $\mathrm{Ne}$ benwirkungen auftraten, ist bis heute im Dunklen geblieben.

Nichtbehandelte depressive Patienten berichteten in einem Drittel der Fälle von Libidorückgang, Ejakulationsverzögerung, Anorgasmie und Erektionsstörungen. Jedoch zeigt ein Anteil von 20\% der z.B. mit 
Clomipramin behandelten Patienten eine weitere Verschlechterung der sexuellen Störung unter der Therapie. Der Zusammenhang zwischen medikamentöser Behandlung und Auftreten der sexuellen Störung ist zwar deutlich, wird jedoch noch ausgeprägter bei Behandlung von Patienten mit Zwangsstörungen, bei denen sexuelle Störungen nicht regelhaft auftreten.

Bei mit Clomipramin behandelten Zwangspatienten treten in bis zu 96\% der Fälle Orgasmusstörungen bis hin zu Anorgasmie auf. Aber nicht nur das Clomipramin, sondern auch andere Tri- und Tetrazyklische Antidepressiva sind mit diesen Nebenwirkungen behaftet. Die Ursache für die sexuelle Funktionsstörung unter dieser Präparategruppe ist die Beeinflussung multipler Neurotransmitter, wie z.B. Serotonin, Noradrenalin, Alpha1-Blockade, muskarinerge und cholinerge Nebenwirkungen. Wichtig erscheinen in diesem Zusammenhang die Untersuchungsdesigns zu sexuellen Störungen unter Medikamenten. Der Unterschied zwischen Nebenwirkungen infolge von Spontanmeldungen versus der Nebenwirkungserfassung durch eine direkte Befragung ist massiv. Bei den SSRI-induzierten sexuellen Störungen betreffen Spontanmeldungen 1 bis 33\% der Patienten, bei direkter Befragung ergeben sich sexuelle Störungen bei bis zu 96\% der Patienten. $\mathrm{Zu}$ therapeutischen Optionen siehe Tabelle 2 .

Andere Antidepressiva: Geringe bis gar keine sexuelle Nebenwirkungen zeigt der MonoaminooxidaseHemmer Moclobemid (6). Vor kurzem erschien sogar eine Studie, in der eine deutliche Verbesserung psychogener Erektionsstörungen bei Patienten ohne depressive Symptome dargestellt werden konnte (7). Ebenfalls seltene sexuelle Funktionsstörungen werden bei Anwendung des Präparates Mirtazapin mitgeteilt.

Andere Indikationen von Antidepressiva: SSRIs und das trizyklische Antidepressivum Clomipramin sind insbesondere wirksam bei zwanghaftem Sexualverhalten, ebenfalls bei Ejakulatio praecox.

Antipsychotika (siehe auch Tab. 3): Traditionell werden hoch- potente von mittel und niederpotenten Antipsychotika unterschieden. Höherpotente Antipsychotika haben eine stärkere antidopaminerge Wirkung, ausgeprägtere extrapyramidale Wirkungen und ebenfalls prolaktinogene Nebenwirkungen. Die Blockade von $\mathrm{D}_{2}$-Rezeptoren führt zu einer Enthemmung (Wegfall der Hemmung durch Dopamin) der Prolaktin-Sekretion. Klinische Effekte beim Mann sind Veränderungen der Appetenz, der Ejakulation, des Orgasmus, Hodenschwellung und Gynäkomastie. Bei Frauen bewirken Prolaktinerhöhungen Brustvergrößerung, Galaktorrhoe, Zyklusunregelmäßigkeiten und Appetenzstörungen, um nur einige zu nennen.

Auch bei den Antipsychotika unterscheiden sich Spontanmeldungen sexueller Nebenwirkungen ausgeprägt von der Erfassung sexueller Nebenwirkungen durch direkte Befragung. Schulze-Mönking erfasste 1996 als Spontanmeldungen 5,5\% gegenüber $19 \%$ sexueller Nebenwirkungen bei direkter Befragung.

Einige atypische Antipsychotika gehen nicht mit Prolaktinerhöhungen einher. Zum ersten ist Clozapin zu nennen, das als Goldstandard der atypischen Antipsychotika genannt werden muss und keinerlei Prolaktinerhöhung bewirkt. Allerdings werden bei diesem Präparat in seltenen Fällen Nebenwirkungen in Form von Priapismus mitgeteilt. Ebenfalls keine wesentliche Prolaktinerhöhung zeigt sich unter dem Präparat Olanzapin. Zwar ist von Beginn der Behandlung bis zu sechs Wochen danach eine Prolaktinerhöhung nachzuweisen, diese fällt nach der sechsten Behandlungswoche jedoch kontinuierlich auf Normalwerte ab. Auch das neue atypische Antipsychotikum Quetiapin weist keine Prolaktinerhöhungen auf. Gelegentliche Prolaktinerhöhungen in bis zu $1 \%$ der Patienten zeigten sich unter $\mathrm{Zi}$ prasidon. Im Gegensatz zu diesen Präparaten werden unter Risperidon und Amisulprid deutliche Prolaktinerhöhungen festgestellt.

Benzodiazepine: Insbesondere unter Alprazolam wurden Libidostörungen und Orgasmusverzögerungen bei bis zu 50\% der Patienten angegeben. Unter Chlordiazepoxid

\section{Tab. 3 Therapeutische Optionen bei sexuellen Nebenwirkungen unter Antipsychotika}

1. Wechsel des Neuroleptikums: Der Wechsel eines Neuroleptikums ist allerdings vorsichtig wegen der hohen Rezidivgefahr durchzuführen. Die Umstellung sollte auf ein atypisches Antipsychotikum mit geringer prolaktinogener Wirkung erfolgen.

2. Dosis-Reduktion: Auch hier besteht die Gefahr der Rezidivprovokation.

3. Bei Hyperprolaktinämie: Zugabe des $D_{2}$ Agonisten Bromocriptin.

4. Zusatzmedikationen: mit Yohimbin, Betanechol und Carbachol.

wurden Orgasmusstörungen und Ejakulationsstörungen gemeldet, wie auch bei Diazepam (11).

Weiterhin sollte das Auftreten sexueller Träume bei Benzodiazepinnarkosen Erwähnung finden. Insbesondere Midazolam und Diazepam sind hier zu nennen. Die Inhalte der Träume können von Betroffenen für reale Erlebnisse gehalten werden, so dass es zu falschen Anschuldigungen von Krankenhausmitarbeitern kommen kann. Die Häufigkeit sexueller Träume bei Benzodiazepinnarkosen wird mit $1: 200$ angegeben.

Eine positive Wirkung können Benzodiazepine bei sexuell bedingten Ängsten entfalten, da hierunter im Einzelfall erst sexuelle Kontakte möglich erscheinen.

Rezidivprophylaktika: Lithium: In der dänischen Studie von Vestergaard und Schou (12) wurden bei rund 10\% der Patienten Libido- und Erektionsstörungen festgestellt. Die Ursache war unklar. Eine noch höhere Rate von Erektionsstörungen zeigte sich in der Studie von Aizenberg (13), hier wurden 20\% dieser sexuellen Funktionsstörungen ermittelt. Falls vertretbar kann die Lithiumdosis reduziert werden.

Carbamazepin: Beobachtet werden hierunter Erektions- und Libidostörungen. Verursacht werden diese vermutlich einerseits durch Prolaktinerhöhungen, andererseits durch Erhöhung des sexual-hormonbindenden Globulins, das zur Reduktion des freien Testosterons führt. Eine therapeutische Option scheint der Wechsel auf das Präparat Oxcarbazepin zu sein. 


\section{Abb.1 Algorithmus zur Diagnostik und Therapie bei substanzinduzierten sexuellen Funktionsstörungen}

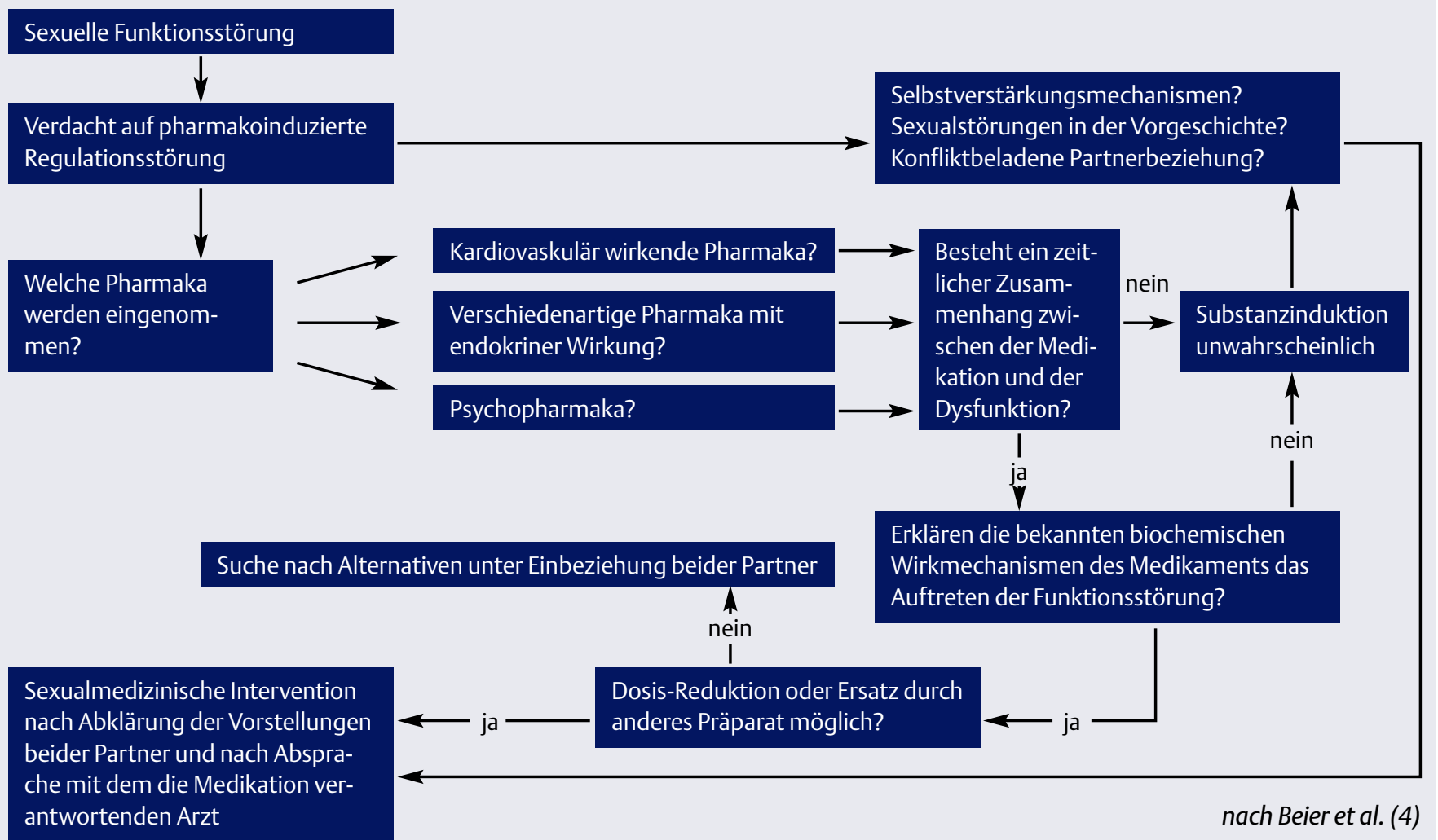

\section{Fazit}

Vielfältige sexuelle Funktionsstörungen können unter Präparaten wie kardio-vaskulär wirksamen Pharmaka und insbesondere Psychopharmaka auftreten. Therapeutische Optionen können Wechsel des Präparates, Reduktion der Dosis, Medikamentenpause oder die Zugabe eines antagonistisch wirksamen Medikamentes sein. Eminent wichtig ist jedoch die Prophylaxe durch die Aufklärung des Patienten und die Abfrage möglicher sexueller Nebenwirkungen bei Medikamentengabe.

\section{Drugs and sexual dysfunctions} Several types of Drugs can produce sexual dysfunctions. Especially cardiovascular, psychotropic drugs and diverse drugs that influence several hormones can produce sexual dysfunctions. Several pharmacological agents and their side effects are discussed, therapeutic opportunities are outlined.

Keywords: sexual dysfunction sexual side effects - psychotropic drugs - cardiovascular drugs

\section{Literatur}

1. Buddeberg C. Sexualberatung. Stuttgart, Enke, 1983

2. Hartlapp J, Zettel S. Sexualstörungen durch Krankheit und Therapie. Ein Kompendium für die ärztliche Praxis. Heidelberg, Springer 1997, 71-79

3. Saß H, Wittchen H-U, Zaudig M. Diagnostisches und statistisches Manual Psychischer Störungen, DSM IV. Göttingen, Bern, Toronto, Seattle, Hogrefe, 1996, 588-592

4. Beier K, Bosinski H, Hartmann U, Loewit K. Sexualmedizin. München, Jena, Urban \& Fischer, 2001, 457-460

5. Labatte LA, Grimes JB, Hines AM, Oleshansky MA. Sexual dysfunction induced by SRIS. Paper presented at: Annual Meeting of the American Psychiatric Association, May 17-22, 1997, San Diego, Calif. Paper Session Abstract 36

6. Philipp M, Baier D. Vergleich der Wirkung von Moclobemid und SSRI auf sexuelle Funktionen bei depressiven Erwachsenen. Psychopharmakotherapie 2000; 7(4): 187-188

7. Mann K, Pankok J, Leissner J. Effects of moclobemide on sexual performance and nocturnal erections in psychogenic erectile dysfunction. Psychopharmacology 2001; 156: 86-91

8. Aizenberg D, Zemishlany Z, Weizman A. Cyproheptadine treatment of sexual dysfunction induced by serotonin reuptake inhibitions. Clin Neuropharmacol 1995; 18 (4): 320-324

9. Balon R. The effects of antidepressants on human sexuality: diagnosis and management. Primary Psychiatry 1995; 2 (8): 46-51 10. Jacobsen FM, Comas-Diaz L. A doubleblind placebo-controlled trial of Yohimbine for treatment of SRI-induced sexual dysfunction. Presented at the American Psychiatric Association 149 th Annual Meeting, New York, May 4-9, 1996, Abstract 716

11. Riley AJ, Riley E J. The effect of single dose diazepam on female sexual response induced by masturbation. Sex Marital Therapy 1986; 1: 49-53

12. Vestergaard P, Poulstrup J, Schou M. Prospective Studies on a Lithium Cohort 3. Tremor, weight gain, diarrhea, psychological complaints. Acta Psychiatrica Scand 1988; 78: 434-441

13. Aizenberg D., Sigler M, Zemishlany Z, Weizman A. Lithium and male sexual function. Clin Neuropharmacol 1996; 19 (6): 515-519

\section{Korrespondenzadresse}

Dr. Uwe Kinzel

Facharzt für Psychiatrie und Psychotherapie Leitender Arzt im Nieders. Landeskrankenhaus Osnabrück

Postfach 2080

49010 Osnabrück 